\title{
LA INFLUENCIA DE LAS TIC EN EL DESEMPEÑO ACADÉMICO: EVIDENCIA DE LA PRUEBA PIRLS EN COLOMBIA 2011 ${ }^{1}$
}

\author{
HÉCTOR A. BOTELLO PEÑALOZA²; ANDREA LÓPEZ ALBA ${ }^{3}$ \\ UNIVERSIDAD INDUSTRIAL DE SANTANDER (UIS)
}

\author{
Recibido, julio 26 de 2014 \\ Concepto evaluación, septiembre 11 de 2014 \\ Aceptado, octubre 10 de 2014
}

\begin{abstract}
Referencia: Botello Peñaloza, H.A.; López Alba A. (2014). "La influencia de las TIC en el desempeño académico: Evidencia de la prueba PIRLS en Colombia 2011". Revista Academia y Virtualidad, 7, (2), 15-26
\end{abstract}

\section{Resumen}

El siguiente artículo calcula el impacto que tienen las tecnologías de la información y comunicación sobre el desempeño lector de niños de cuarto de primaria, utilizando la prueba Progreso Internacional en Competencia Lectora (PIRLs) de 2011 para una muestra de alumnos en Colombia. Para la estimación se utilizaron técnicas de regresión lineal múltiple, ya que permite el control de variables institucionales, familiares e individuales, para así hallar el efecto directo que tienen las TIC en el puntaje promedio del estudiante. Los resultados muestran que la tenencia de tecnologías y el uso de éstas en el aprendizaje escolar mediante actividades de lectura de contenido digital, afectan positivamente el desempeño lector de los niños, incrementando el puntaje promedio entre un $21 \%$ y un $31 \%$. Asimismo, el uso de computador (PC) para efectos de mejorar la lectura aporta un 32\% adicional en la calificación de la prueba respecto a los niños que no usan PC para esto, siendo la variable de mayor peso. No obstante, se hace énfasis en el uso adecuado de las TIC, ya que su utilización llana tanto en el hogar como en el colegio puede afectar negativamente el desempeño dado que se utilizan para otros objetivos fuera del escolar.

Palabras clave: Colombia, desempeño lector, educación primaria, PIRLS, TIC.

\section{THE INFLUENCE OF TICS ON ACADEMIC PERFORMANCE: EVIDENCE FROM PIRLS TEST IN COLOMBIA 2011}

\section{Abstract}

This paper estimated the impact that have the technologies of information and communication on the reader performance of children of fourth primary, using 2011 reading competition (PIRLS) international progress test to a sample of students in Colombia. Multiple linear regression techniques were used to estimate since it allows the control of insti-

1. Artículo de investigación, resultado de la línea Desarrollo Regional, Grupo de Investigación Desarrollo Regional y Ordenamiento Territorial durante 2014, y apoyo de la uIs.

2. Maestría Ingeniería Industrial (en curso), Universidad Industrial de Santander (UIS); Profesor Política Monetaria y Fiscal (UIS). Correo: hectoralbertobotello@gmail.com

3. Estudiante Economía Universidad Industrial de Santander (UIS). Correo: andrealopezalba@gmail.com 
tutional, individual, and family variables to find the direct effect that TIC have on the average score of the student. The results show that possession of technologies and the use of these in school learning through reading digital content, positively affect the reader performance of children, increasing the average score between $21 \%$ and $31 \%$. Also, the use of the PC to improve the reading contribute with additional $32 \%$ in the test qualification in relation with the children that doesn't use a PC. However, goes emphasis on the proper use of the TICs, since the full use both at home and in school can adversely affect the performance since they are used for other purposes outside the school.

Keywords: Colombia, reader performance, primary education, PIRLS, TIC.

\section{A INFLUÊNCIA DAS TIC NO DESEMPENHO ACADÊMICO: EVIDÊNCIA DA PROBA PIRLS NA COLÔMBIA 2011}

\section{Resumo}

Neste artigo calcula-se o impacto que têm as tecnologias da informação e comunicação sobre o desempenho leitor nas crianças de quarto de primaria, com a aplicação da proba Progresso Internacional na Competência Leitora (PIRLS) de 2011 para uma amostra de alunos na Colômbia. Para a estimação foram utilizadas técnicas de regressão linear múltipla, uma vez que elas permitem o controle de variáveis institucionais, familiares e individuais, para assim achar o efeito direito que têm as TIC na pontuação media do estudante. Os resultados mostram que a possessão de tecnologias e o seu uso no aprendizado escolar mediante atividades de leitura com conteúdo digital, afetam positivamente o desempenho leitor das crianças, incrementando a pontuação media entre um $21 \%$ e um 31\%. Assim mesmo, o uso de computador (PC) com a finalidade de melhorar a leitura aporta um 32\% adicional na qualificação da proba respeito de crianças que não usam PC para isso, sendo a variável de maior peso. Embora, a ênfase se faz no uso adequado das TIC, uma vez que sua simples utilização tanto no lar quanto na escola pode afetar negativamente o desempenho pois são utilizados para outros fins fora do escolar.

Palavras-chave: Colômbia, desempenho leitor, educação primaria, PIRLS, TIC.

\section{Introducción}

La calidad de la educación en Colombia ha sido puesta a prueba en diferentes ocasiones gracias a la implementación de diferentes pruebas internacionales. El resultado, un desempeño consistentemente bajo en comparación con la media internacional (Banco Mundial, 2009). Con el ánimo de mejorar la calidad educativa del país, los últimos esfuerzos de la política pública ha sido la implantación de programas orientados a la puesta en marcha de una educación cada vez más globalizada, en la que los estudiantes utilicen las tecnologías de la información y la comunicación (TIC) para mejorar su rendimiento académico (Castells, 1999).

La idea anterior se basa en el supuesto que el desempeño escolar de los niños está determinado por la interacción de los ámbitos sociales, familiares e individuales de los estudiantes. En este sentido, diversos estudios (Aypay, 2010; Meelissen y Drent, 2008; Biagi y Loi, 2013) han mostrado que los instrumentos y herramientas utilizadas dentro y fuera del contexto educativo son artífices significativos en la mejora en el desempeño de los infantes. Por esto, las tecnologías de la información y la 
La influencia de las tic en el desempeño académico: Evidencia de la prueba pirls en colombia 2011

comunicación (TIC) sobresalen, gracias a su capacidad de manejar eficientemente la información, generando externalidades positivas entre individuos, incrementando las redes del conocimiento y el aprendizaje (Borthwick y Lobo, 2005).

Según Ferro et ál. (2009), una consecuencia de la implementación de las TIC en el aprendizaje es la eliminación de las barreras espacio-temporales a las que se ha visto condicionada la enseñanza presencial. Desde este punto de vista, se asume que el aprendizaje ya no se produce en un espacio netamente físico sino digital, el ciberespacio, en el cual se tienden a desarrollar interacciones comunicativas mediáticas. De este modo, las instituciones educativas pueden realizar ofertas de cursos virtuales, posibilitando la extensión de sus estudios a colectivos que -por distintos motivos-no pueden acceder a las aulas, cursándolos desde cualquier lugar.

Esta flexibilidad de la puesta en marcha de diferentes cursos ha conducido a la denominada educación bajo demanda, la cual consiste en responder a las necesidades de formación concretas de los individuos. Asimismo, ha permitido que el alumnado sea más consciente con su proceso de aprendizaje al poder seleccionar y organizar su currículo formativo (Salinas, 1998; 1999); hecho que permite la expansión de la educación centrada en el estudiante no en el docente.

Pero cuando se examina la relación de estos dos agentes, se puede aceptar que las TIC también han mejorado la comunicación entre los distintos agentes del proceso enseñanza-aprendizaje, en especial entre estudiantes y docentes, incrementando los flujos de información y la colaboración entre ellos más allá de los límites físicos y académicos de las instituciones de enseñanza. Por ejemplo el envío de consultas o trabajos de los estudiantes a sus docentes desde cualquier lugar y en cualquier momento (Marqués, 2001: 92). En este mismo sentido, permite facilitar la organización de actividades grupales entre los mismos estudiantes (Cenich y Santos, 2005). En definitiva, el uso de las TIC, mejora la eficacia educativa al poder desarrollarse nuevas metodologías didácticas que benefician el proceso de formación del estudiante.
Además, ofrecen un mayor impacto para el desarrollo de sus habilidades. Por este tipo de particularidades, las TIC se han ido incorporando rápidamente en los procesos de enseñanza-aprendizaje educativa (Cooperberg, 2002), y consecuentemente ha conducido a la informática educativa.

Este artículo, de hecho, estima el impacto de las TIC en el desempeño académico de los estudiantes Colombianos a partir de los datos de la prueba PIRLS del año 2011, con la implementación de modelos de regresión lineal múltiple. Para lograr lo anterior, en la sección siguiente se desarrolla el marco teórico de los conceptos que envuelven a las TIC y el desempeño académico exponiéndose la literatura consultada. En la tercera sección se presenta la descripción de la metodología y posteriormente, en la cuarta sección, se enumeran los resultados de la investigación relacionándolos con otros encontrados en la literatura. Para finalizar, en la quinta parte se exponen las conclusiones y, seguidamente, se presenta la bibliografía investigada.

\section{Marco teórico}

En los trabajos empíricos que analizan el impacto del uso de las TIC sobre el desempeño escolar, en general, se realizan comparaciones cualitativas entre las variables asociadas y son pocos los estudios en los que se encuentra un carácter cuantitativo. Esto se debe, tal y como lo afirman Biagi y Loi (2013), a que el cálculo de la influencia que ejercen las TIC en el desempeño escolar es un problema difícil de estudiar debido a los innumerables factores observables y no observables que inciden, los que se exponen en la figura 1.

En este sentido, los estudios revisados llegan a resultados variados y algunos de éstos han encontrado una evidencia moderada sobre el rendimiento académico de los estudiantes que emplean las TIC, otras veces una efectividad mínima y otras, ninguna. Entre éstos se encuentran el de Wengslinky (1998), que -utilizando una prueba nacional de conocimiento en Estados Unidosindica que la tecnología puede hacer una diferencia pero depende de la forma como se aplica. 
Por otro lado, Fuchs y Woessman (2004), con base en los resultados de PISA 2003, encuentran que existe una relación negativa entre el rendimiento académico de los estudiantes y el uso del computador en la casa, y que con respecto al uso del computador en el colegio no hay una clara tendencia que mejore el desempeño académico.

Otroejemplo, dentro de los estudios netamente cualitativos, Meelissen y Drent (2008) encuentran en una muestra de estudiantes holandeses que la intensidad del uso de la TIC con fines educativos mejora el desempeño escolar. En cambio, entre estudiantes turcos, Aypay (2010) no encontró una relación significativa entre el uso de TIC y el desempeño académico porque las tecnologías se emplean con mayor frecuencia en aspectos de entretenimiento y diversión en internet. Este último resultado es similar al del estudio cuantitativo de Goolsbee y Guryan (2006) donde no encuentran evidencia de un mejor rendimiento en pruebas académicas de Estados Unidos aunque el uso de las TIC vayan en aumento; sin embargo, señalan que hay efectos positivos en los resultados de aprendizaje de los estudiantes, pero limitados al ámbito de interés o una asignatura particular.

En otras variables importantes que consideran algunos estudios, está la formación, capacitación y utilización que tenga el profesorado de las TIC en la clase, así como con la intensidad de su uso (Meelissen y Drent, 2008; Spiezia, 2010) tanto dentro como fuera de la escuela (Spiezia, 2010). Entre los trabajos que encuentran relaciones positivas al introducir estos factores se ubica el de Notten y Kraaykamp (2009), quienes encuentran una relación positiva de tales factores sobre el desempeño escolar en distintas pruebas aplicadas y con énfasis en ciertas asignaturas. Un resultado contrario fueron las investigaciones de Angrist y Lavy (2002) que muestran que, al controlar las características observables, el mayor uso educativo de los ordenadores no tiene efecto positivo en las pruebas estandarizadas; estos dos últimos estudios aplicados sobre la misma base de información, la prueba PISA 2006.
Otras variables de control tal como el género, la edad y la etnia, son significativas en varios análisis. Los resultados de Makrakis y Sawada (1996) señalan que las niñas poseen menos actitud positiva hacia las TIC que el género de su contraparte, tanto en la escuela como en casa, y por ello obtienen puntajes menores en las pruebas para medir el conocimiento y las destrezas. Los estudios de Comber, Colley, Hargreaves y Dorn (1997) sugieren que los estudiantes de grados mayores poseen una actitud media hacia la TIC como los de grados menores. Por su parte, los estudios de Volman, Van Eck, Heemskerk y Kuiper, 2005 ) indican que 'los de color' (minoría de la población) se consideran menos capaces, con restringido acceso y con distintos objetivos para uso de las TIC que la mayoría de la población.

Al final, el pensamiento que queda después de la revisión es el mismo que el de McFarlane, Sparrowhawk y Heald (2002), quienes comentan que nunca se debe olvidar que las TIC son un instrumento y, por lo tanto, sus resultados dependen del uso que se haga de ellas. En este orden de ideas, este trabajo busca contribuir desde un punto de vista cuantitativo, investigando únicamente sobre un área del conocimiento como es la del lenguaje en individuos que se supone han formado un marco cognoscitivo para esta actividad como son los niños de cuarto grado de primaria, en tanto que saben leer.

Bajo este esquema de pocos estudios de carácter cuantitativo, surge la inquietud de realizar estudios que midan el impacto de estas tecnologías en el proceso educativo, con especial énfasis en países en desarrollo, donde una mayor calidad educativa es el camino para lograr un mayor desarrollo económico y, a la vez, constituye un reto en tanto la inversión que destinan para ello. 


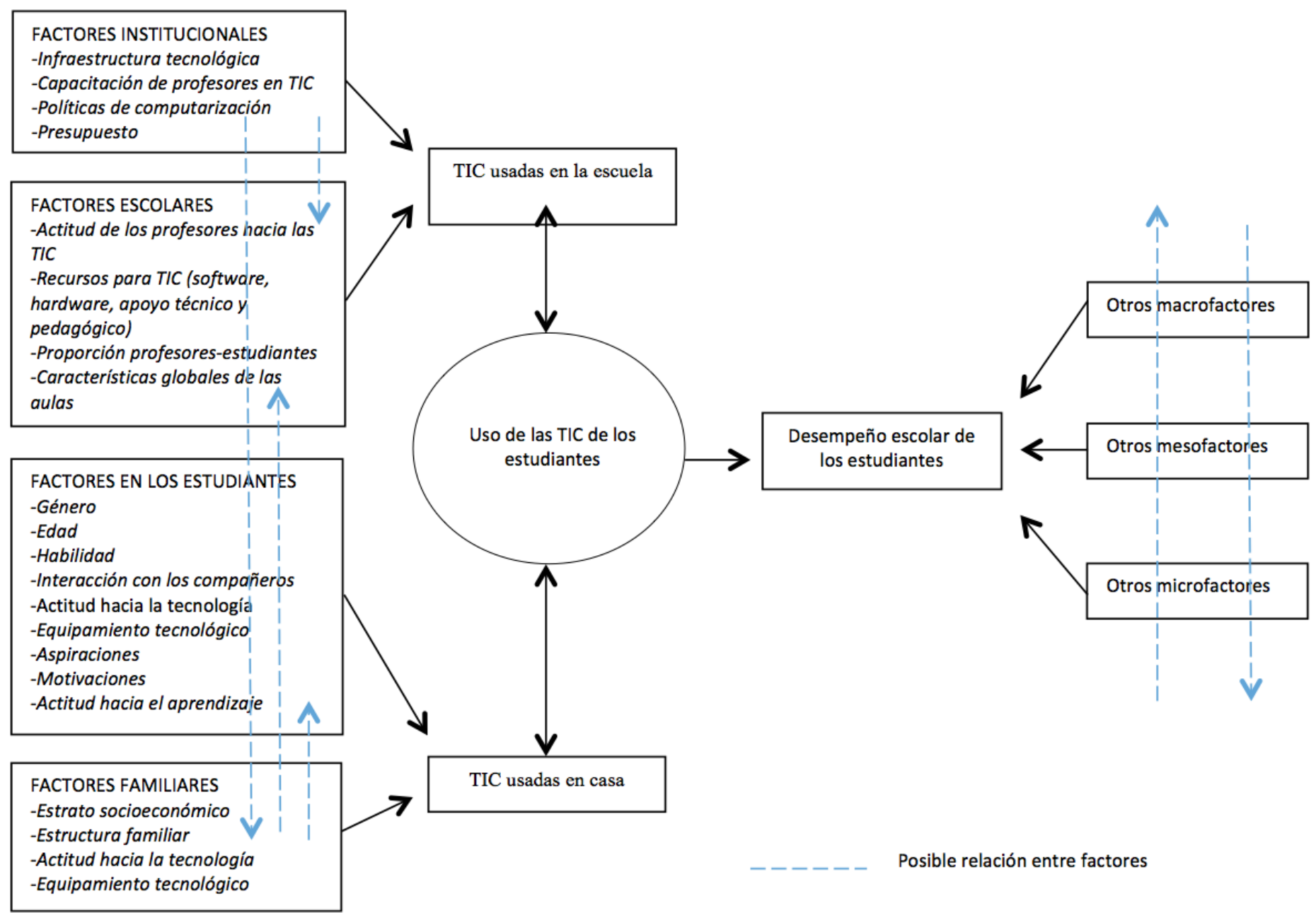

Figura 1. Variables consideradas factores del rendimiento escolar 2013 (traducción de los autores).

\section{Metodología}

A continuación se resume el modelo lineal múltiple y la fuente de datos utilizados para la medición de la influencia de las TIC en el desempeño lector de los niños de cuarto de primaria en Colombia.

\section{Fuentes de datos}

Se utilizó la base de datos del estudio sobre el Progreso Internacional en Competencia Lectora (PIRLS) del año 2011, extrayendo únicamente los datos pertinentes para Colombia. Esta medición, junto con otras como First International Mathematics Study (FIMS), Second
International Mathematics Study (SIMS), Second International Science Study (SISS), Third International Mathematics and Science Study (TIMS), son dirigidas por la Asociación Internacional para la Evaluación del Rendimiento Educativo (IEA) que, desde 1960, con una prueba de matemáticas aplicada a doce países, emprende este tipo de mediciones a nivel internacional, haciendo la salvedad que las metodologías han cambiado con el tiempo.

Esta prueba considera que la competencia lectora de los infantes está condicionada por la motivación que se tiene para leer y distingue dos propósitos, según el Instituto Nacional de Evaluación Educativa de España:

- La lectura como experiencia literaria (generalmente realizada para disfrute personal). 
- La lectura para adquisición y uso de información, realizada como parte de la interacción

social o como instrumento para el aprendizaje.

Según el manual metodológico de PIRls (Foy, 2013), la prueba se realiza con estudiantes de cuarto grado de primaria en 56 países. Se realiza mediante un cuadernillo de preguntas que incluye dos bloques de evaluación, para los que el alumno dispone de dos períodos de 40 minutos, separados por un descanso de 20 minutos.

Cada bloque de evaluación consta de un texto de estímulo de aproximadamente de 900 palabras, seguido de las preguntas de varios tipos: opción múltiple, abierta o de respuesta construida. La mitad de los textos son literarios (pequeños relatos o cuentos), y la otra mitad son informativos en forma de artículos infantiles sobre un tema, un folleto de excursión, etc.

En ésta también se aplican cuestionarios de contexto o de factores asociados, dirigidos a estudiantes, a padres de familia, a docentes y a rectores de las instituciones educativas; además, recoge información sobre políticas educativas de los países participantes para conocer aspectos de los contextos en los cuales se desarrolla la competencia lectora de los niños.

En relación con el análisis de los resultados, IEA desarrolla la Enciclopedia PIRLs, la cual consiste en una publicación que proporciona datos de todos los países participantes, fundamentalmente relativos al sistema educativo $\mathrm{y}$, en particular, al currículo de la educación primaria y el lugar que en él ocupa la enseñanza de la lectura. Los datos de la Enciclopedia se obtienen mediante un cuestionario a las autoridades sobre el currículo escolar y cómo se contempla en él la enseñanza de la lectura.

Dentro de esta prueba se escogieron varias preguntas para aproximar el uso de TIC (variables proxy) por parte de los escolares, siendo éstas:
- Tenencia de computador (PC) en el hogar.

- Tenencia de conexión a internet en el hogar.

- Frecuencia de uso PC en el hogar (veces por mes)

- Frecuencia de uso PC en el colegio (veces por mes)

- Uso del PC para búsqueda de información, lectura, redacción y otros software utilizados en la clase de lenguaje.

\section{Número de computadores por alumno de 4 grado en el colegio.}

\section{Modelo}

En este trabajo se supone que el desempeño lector de los estudiantes de cuarto primaria está determinado por factores familiares, escolares, individuales y por el uso de las TIC. En este orden de ideas, el puntaje en la prueba de lectura será la variable dependiente mientras que las variables socioeconómicas (familia, escuela, contexto) serán las independientes; junto con las que se relacionen de manera lineal con el uso de las TIC.

De manera econométrica se podría definir la siguiente función logarítmica:

$$
y=\alpha+\beta_{1} F+\beta_{2} E+\beta_{3} I+\beta_{4} T I C+\varepsilon
$$

Donde Y es el logaritmo del puntaje ${ }^{4}$ en lectura de la prueba PIRLS de los niños de cuarto grado, mientras que I,E,F y TIC representan los conjuntos de variables asociados a cada área. Por su parte, $\beta_{\mathrm{i}}$ representan los vectores de coeficientes que relacionan los impactos de cada una de las variables independientes sobre la independiente. Finalmente, $\varepsilon$ es el error del modelo, el cual se divide mediante una distribución normal con media cero y varianza constante.

Para la estimación se utiliza la técnica de regresión lineal múltiple que permite el control de variables socioeconómicas, familiares y escolares de los individuos. La forma de estimación es mediante el uso de los mínimos

4. Por cuestiones de estimación en relación a la reducción de la varianza de la muestra se realizó una transformación lineal de la variable dependiente. 
La influencia de las tic en el desempeño académico: Evidencia de la prueba pirls en colombia 2011

cuadrados ordinarios; para así encontrar el efecto directo que tienen las TIC en el desempeño lector de los estudiantes. La efectividad de este modelo se evalúa mediante tres criterios. El primero de ellos, R2, mide el porcentaje de la varianza de la variable dependiente captada por parte de las independientes. En segunda instancia, se comprueba si el modelo en su conjunto es significativo al querer explicar la variable dependiente mediante el indicador del F de ajuste global, entre más alto, más probable es rechazar la hipótesis nula de no significancia conjunta del modelo a través de valores más altos de este indicador. En tercer lugar, se prueba la significancia individual de las variables dentro del modelo, observando el valor de la probabilidad menor al 5\% para rechazar la hipótesis de nulidad.

Las variables de control serán las características del niño (ej. sexo, edad, edad en la que entró a preescolar), del hogar (supervisión de los padres sobre las tareas, tiempo de lectura en casa, número libros en casa, ocupación de los padres, educación de los padres, población en el área, nivel de ingreso de la zona donde habita el alumno, país donde habita) y del colegio (número de profesores, años de experiencia del profesor, sexo del profesor, edad del profesor, educación del profesor, área donde queda del colegio, tamaño del colegio, tamaño del salón de $4^{\circ}$ grado.).

Las variables de tratamiento serán las variables que se mencionaron anteriormente que se relacionan con el uso de TIC. En este sentido, después de la elección de las variables y la depuración de la base de datos de las observaciones no válidas para el análisis, las estimaciones se realizaron sobre 3.460 estudiantes.

\section{Resultados y análisis}

En PIRLS 2011, en general, participaron cerca de 325.000 estudiantes. Las muestras representativas de cada país estuvieron conformadas por aproximadamente 4.000 alumnos dentro de un rango de 150 a 200 establecimientos educativos. Por Colombia participaron 3.966 alumnos de 150 establecimientos educativos oficiales y privados, urbanos y rurales, de ambos calendarios académicos.
Entre los resultados generales, Colombia posee un desempeño lector medio de 448 puntos e inicialmente no se aprecia una relación directa entre la utilización de las TIC en el aula y el puntaje promedio obtenido en la prueba, tal y como lo muestra la figura 2.

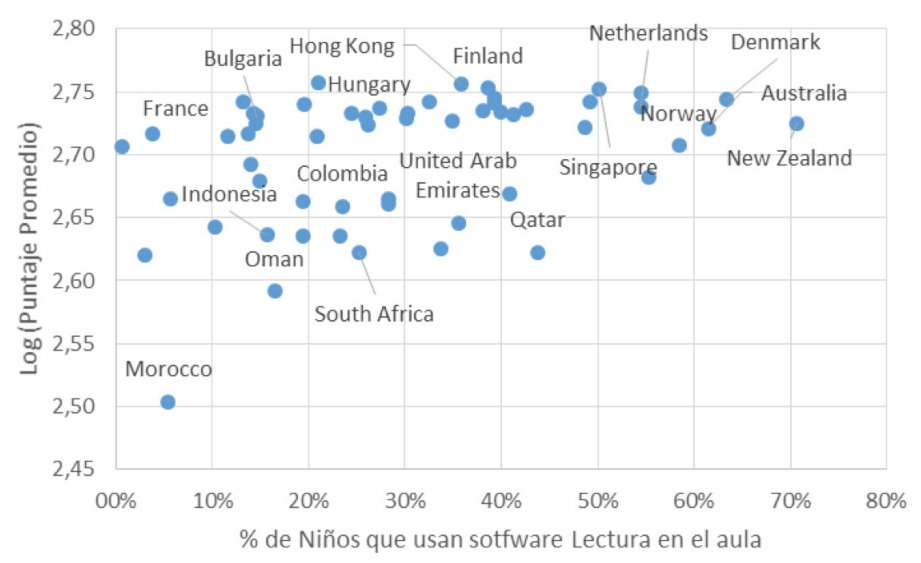

Figura 2. Estudiantes que usan software de lectura versus puntaje promedio PIRLS 2011 por país

Fuente: Asociación Internacional para la Evaluación del Rendimiento Educativo (IEA), Prueba PIRLS 2011

Asimismo, se encuentran grandes disparidades en el acceso y tenencia de TIC. En este sentido, el 93\% de los colegios investigados declararon tener infraestructura computacional, pero sólo el $43 \%$ de éstos los utilizan activamente en la enseñanza de lectura. Colombia se encuentra sobre la media de los países investigados con el $69 \%$ de los estudiantes, utilizando ordenadores para actividades de lectura en el colegio, tal como lo muestra la siguiente figura.

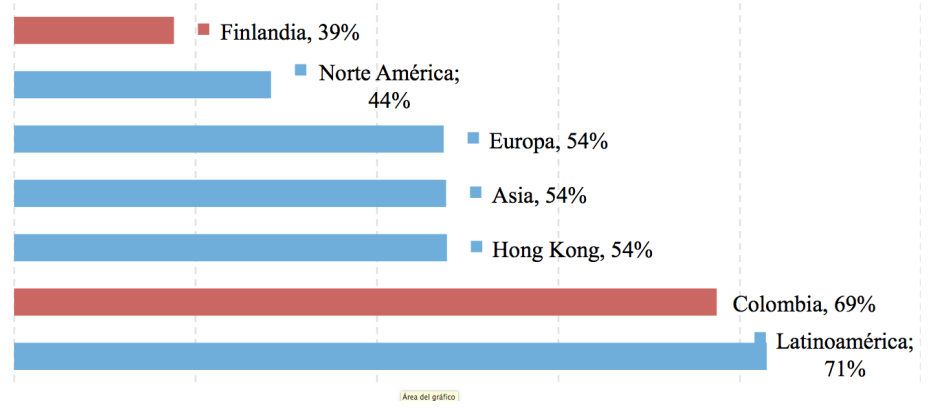

Figura 3. Porcentaje de estudiantes que usan el PC para actividades de lectura en el colegio

Fuente: Asociación Internacional para la Evaluación del Rendimiento Educativo (IEA), Prueba PIRLS 2011 
Héctor A. Botello Peñaloza ; Andrea López Alba

A continuación se aplica la ecuación 1 con las variables seleccionadas sobre los datos disponibles para Colombia en el año 2011. Los resultados de la estimación son presentados en la tabla 1 donde se muestran las variables tanto continuas como categóricas, sus características y el efecto porcentual que tienen sobre el puntaje.

En este caso, el efecto de las variables continuas se interpreta como el cambio porcentual que tendrá el puntaje con una variación del $1 \%$ de la variable independiente. Por ejemplo, un aumento del $1 \%$ en la relación de computadores por alumno de cuarto grado en determinado colegio colombiano, aumenta en un $0,1 \%$ el puntaje promedio en la prueba de lectura. Por otro lado, en las variables categóricas, el cambio porcentual sobre el puntaje se da con relación a una característica base. Por ejemplo, la no tenencia de PC en la casa del niño disminuye en un $4,93 \%$ su puntaje en comparación con los que sí tienen PC.

En términos generales, las variables de la tabla 1 son todas significativas al 5\% de confianza, con un R cuadrado del 0.53 . Esto significa que cerca del $53 \%$ de la varianza total del puntaje de la prueba PIRLS está siendo captado o explicado por las variables dentro del modelo, por lo que las conclusiones que se obtengan son válidas para la muestra seleccionada.

En relación con las variables que se utilizaron como proxy de la implementación de TIC, éstos fueron los efectos encontrados:

- Tenencia de internet en el hogar: La tenencia de una conexión a internet en los hogares presenta un puntaje promedio en la prueba mejor en 1,3\% que los que no tienen la conexión.

\begin{tabular}{|c|c|c|c|}
\hline Variable & Característica & Coeficientes & Características Base \\
\hline \multicolumn{4}{|l|}{ Variables Asociadas a las TIC } \\
\hline \multirow[t]{2}{*}{$\log \left(N^{0} P c\right.$ por alumno 4 en el colegio $)$} & & 0.1062 & Continua \\
\hline & & $(0.0146)$ & \\
\hline \multirow[t]{2}{*}{ Posee PC en casa } & No & -0.0493 & Sí \\
\hline & & $(0.0117)$ & \\
\hline \multirow{2}{*}{ Internet en la casa } & No & -0.013 & Sí \\
\hline & & $(0.0006)$ & \\
\hline \multirow[t]{6}{*}{ Frecuencia lectura PC en colegio } & Una o dos veces por semana & -0.2411 & Todos los días \\
\hline & & $(0.0427)$ & \\
\hline & \multirow[t]{2}{*}{ Una o dos veces por mes } & -0.2198 & \\
\hline & & $(0.0443)$ & \\
\hline & \multirow{2}{*}{ Nunca o casi nunca } & -0.3183 & \\
\hline & & $(0.0536)$ & \\
\hline \multirow[t]{3}{*}{ Frecuencia uso PC en casa } & Una o dos veces por semana & 0.0164 & Todos los días \\
\hline & & $(0.0097)$ & \\
\hline & Nunca o casi nunca & 0.0487 & \\
\hline \multirow[t]{6}{*}{ Frecuencia uso PC en colegio } & Una o dos veces por semana & 0.0692 & Todos los días \\
\hline & & $(0.0116)$ & \\
\hline & Una o dos veces por mes & 0.047 & \\
\hline & & $(0.0153)$ & \\
\hline & Nunca o casi nunca & 0.0409 & \\
\hline & & $(0.0135)$ & \\
\hline Variables asociadas al estudiante & & & \\
\hline Edad del estudiante & 8 años & -0.3039 & 13 \\
\hline & & $(0.0342)$ & \\
\hline & 11 años & 0.0156 & \\
\hline & & $(0.0078)$ & \\
\hline Posee libros en casa & No & -0.0543 & Sí \\
\hline & & $(0.0096)$ & \\
\hline Educación Padres & Primaria & -0.038 & Universitario \\
\hline & & $(0.0094)$ & \\
\hline & Ninguna & -0.1999 & \\
\hline & & $(0.0598)$ & \\
\hline Ocupación de los padres & Administrativo & -0.0175 & Profesional \\
\hline & & $(0.0096)$ & \\
\hline & Trabajador Cualificado & -0.0323 & \\
\hline & & $(0.012)$ & \\
\hline Tiempo de lectura fuera del colegio & 30 minutos a una hora & 0.0369 & Menos de 30 minutos \\
\hline & & $(0.0083)$ & \\
\hline & 1 hora a 2 horas & 0.0265 & \\
\hline & & $(0.012)$ & \\
\hline Variables asociadas al colegio & & & \\
\hline Género del docente & Hombre & -0.1005 & Mujer \\
\hline & & $(0.0171)$ & \\
\hline Edad del docente & 40 a 49 años & -0.1551 & Menores a 25 años \\
\hline & & $(0.0131)$ & \\
\hline & 50 a 59 años & -0.1506 & \\
\hline & & $(0.0162)$ & \\
\hline Localización del colegio & Cabecera dispersa & -0.0531 & Urbano \\
\hline & & $(0.0146)$ & \\
\hline & Rural & -0.2055 & \\
\hline & & $(0.0328)$ & \\
\hline Librería en el colegio & No & -0.0584 & Sí \\
\hline & & $(0.0129)$ & \\
\hline Log (Alumnos Totales 4 grado) & & -0.0424 & \\
\hline & & $(0.015)$ & \\
\hline Log (Alumnos Totales del colegio) & & -0.0168 & \\
\hline & & $(0.0062)$ & \\
\hline Población de la ciudad & 50 mil a 100 mil personas & 0.106 & Más de 500 mil \\
\hline & & $(0.0199)$ & \\
\hline & $15 \mathrm{mil} \mathrm{a} 50 \mathrm{mil}$ & 0.0428 & \\
\hline & & $(0.0146)$ & \\
\hline & menos de 3 mil & 0.1287 & \\
\hline & & $(0.024)$ & \\
\hline Constante & 6.3778 & vaciones & 3.460 \\
\hline & $(0.0445)$ & Ajustado & 0.5384 \\
\hline
\end{tabular}

Tabla 1. Resultados de las estimaciones (errores estándar entre paréntesis)

Fuente: Cálculos de los autores con base en la información de la Asociación Internacional para la Evaluación del Rendimiento Educativo (IEA), Prueba PIRLS 2011 
La influencia de las tic en el desempeño académico: Evidencia de la prueba pirls en colombia 2011

- El uso del PC para lectura: Los niños que en la clase a diario realizan sus actividades lectoras y de redacción a través de medios digitales mostraron en promedio un $24 \%$ adicional en su puntaje de lectura que los niños que lo hacen uno a dos veces por semana. Un $22 \%$, si se compara con los de frecuencia de una o dos veces por mes. Y un 32\% comparado con los que no hacen esto. Este efecto es el mayor factor que determina el desempeño lector de los niños de cuarto de primaria en Colombia.

- Frecuencia de uso de PC en el colegio y el hogar: El uso diario del equipo informático en el colegio puede representar hasta entre un $4 \%$ y $6 \%$ superior en el puntaje promedio respecto a los niños que lo usan con una frecuencia semanal o menor. Contrariamente, el uso de PC en el hogar con la frecuencia anterior mencionada, tiende a disminuir entre el 5\% y el 1,62\% el puntaje de la prueba de lectura.

El conjunto de los resultados de la presente investigación muestra un impacto positivo sobre el desempeño lector de los niños causado por las TIC; resultados que son contrarios a los hallados por Fuchs y Woessman (2004), para el caso estadounidense en el año 2003, y por la OCDE (2010) en la cual se estableció, además de una relación negativa entre las TIC y el rendimiento académico, un mayor uso de estas tecnologías en el hogar que en los establecimientos educativos. No obstante, el efecto no es el uso indiscriminado de las tecnologías sino el uso orientado hacia la actividad lectora.

Otros resultados relevantes de las estimaciones muestran que los niños que poseen libros en la casa pueden reportan un puntaje hasta un 5\% mayor que su contraparte. Este efecto se ve multiplicado si se tiene en cuenta el tiempo de lectura de los niños. Cuando éstos leen de 30 minutos a una hora o de una hora a 2 horas, pueden ganar adicionalmente entre un $3,7 \%$ y un $2,65 \%$ respectivamente.

No obstante, este último efecto puede estar determinado al mismo tiempo por la educación de los padres. En la estimación se observa cómo los hijos de padres con grados universitarios pueden obtener hasta un $4 \%$ más de rendimiento que los padres con grados de primaria y hasta un $20 \%$ de los que no tienen ningún grado educativo. Esto puede deberse a que los "padres más educados" en cuanto a su nivel educativo, tienden a educar a sus hijos de la misma manera y a inculcar en ellos hábitos cognitivos tales como leer y escribir.

El anterior efecto es estudiado ampliamente en la literatura. Muestra de ello son los estudios de Gorman y Politt (1999); Sutton y Soderstrom (1999) y Buckner, Bassuk y Weinreb (2001). Sobre la teoría se argumenta que el desempeño académico de los niños está mediatizado por el nivel cultural de las familias de procedencia y éste, a su vez, por el ambiente familiar relativo a las actitudes, expectativas y valoración de la familia con respecto al desarrollo académico de los niños. A la vez, este fenómeno se correlaciona con el nivel socioeconómico del hogar, medido por los ingresos del mismo. La correlación es positiva, a medida que se tienen ingresos mayores en familias "educadas", se tendrán mayores incentivos para invertir en la educación de los hijos.

Otra forma de mostrar lo anterior, es observar dónde se localiza el colegio de los estudiantes. Se aprecia cómo los colegios ubicados en las áreas de la cabecera y en el sector rural, presentan puntajes de un 5\% y $20 \%$ menos que los localizados en el sector urbano. Sin embargo, un aspecto interesante es que los colegios localizados en ciudades pequeñas pueden generar mejores resultados. En este sentido, los estudiantes que reciben sus clases en ciudades con menos de tres mil habitantes obtienen en promedio puntajes $12 \%$ mayores que los localizados en ciudades con más de 500 mil personas. Esta situación es similar en ciudades de 50 mil a 100 mil personas pero con el 10\%.Sin embargo, no se deben dejar de lado aspectos directos al colegio y a la contratación de maestros, tales como la edad y el género del profesor. Se aprecia que los estudiantes de cuarto grado de docentes menores de 25 años, logran tener puntajes $15 \%$ mayores que sus pares con mayor edad. En este mismo sentido, se observa que los estudiantes de cuarto grado con docentes de sexo femenino, tienen puntajes $10 \%$ mayores que cuando sus docentes son de género masculino. 
En línea con el tamaño del curso y del colegio, las estimaciones muestran que un aumento del $1 \%$ del número de estudiantes de 4 grado del colegio, disminuye en un $0.04 \%$ el puntaje de la prueba lectora. Mientras que un aumento del $1 \%$ en el número de estudiantes totales del colegio, baja en un $1.68 \%$. Estos resultados son acordes a los alcanzados por Legler, Licht y Spielkamp (2000). El fenómeno en el que salones de estudio más pequeños logran mejores desempeños, se logra gracias a que un menor número de alumnos en una clase permite que el número de interrupciones y de ruido en el aula disminuyan, permitiendo al docente realizar otro tipo de actividades pedagógicas en el aula. Asimismo, con grupos de clase pequeños, el docente puede brindar una atención más personalizada y centrarse en las necesidades específicas de cada estudiante en lugar de las del grupo como un todo. Además, el tamaño del grupo afecta el nivel de ajuste o acoplamiento de los menores al mismo; a menor número de estudiantes se pueden llevar cabo actividades que exijan la participación de cada individuo (Lee y Wu, 2012).

\section{Conclusiones}

La mayor parte de los estudios revisados muestran que las TIC pueden ser utilizadas para incrementar el desempeño lector de los niños de cuarto primaria. Es así, como este trabajo muestra que no es sólo la presencia de las TIC la que determina un mayor desempeño de los estudiantes, sino su utilización efectiva dentro del aula la que permite sostener esta tendencia, medida por el género del docente, la edad del mismo, el tamaño del grupo y del colegio, la ubicación geográfica del colegio, el nivel educativo de los padres, entre otros.

En Colombia, los resultados muestran que el uso conjunto de las TIC permite incrementar el puntaje promedio de los niños de cuarto grado en la prueba lectora PIRLS entre un $21 \%$ y un $31 \%$. Este resultado se obtuvo después de controlar diversas variables institucionales, familiares e individuales, siendo el mayor efecto cuando las TIC se utilizan enfocadas a actividades de lectura dentro del aula. Las implicaciones del anterior resultado sobre los programas de calidad educativa son principalmente dos: la primera, no sólo es la dotación de infraestructura tecnológica educativa la que por sí solo traerá una mayor calidad en la prueba lectora, sino que también, y ésta es la segunda idea, se hace necesaria la capacitación de los docentes en el uso activo de estas tecnologías dentro de los programas educativos, es decir que al mismo tiempo en el que se dota a las escuelas y estudiantes con mayores herramientas y tecnologías, se debe invertir una mayor cantidad de tiempo capacitando al personal docente para que pueda exponer a los estudiantes todo el material informático que pueden proveer las TIC.

Conforme a los resultados encontrados, resulta importante resaltar la pertinencia del uso de tecnologías de información y comunicación como una herramienta para lograr mayores puntajes promedios en la prueba PIRLS, según las especificaciones mencionadas anteriormente, tales como la capacitación del personal docente en cuanto al uso y los beneficios de ésta en las instituciones educativas, así como que los padres sean conscientes de la práctica que se le dan a las TIC y los fines para los que se usan, en los hogares. En este sentido, las TIC hacen parte de una aproximación al mejor desempeño académico de los alumnos motivado por el uso de PC para fomentar actividades que promuevan el aumento de capacidades para la lectura, así como usarlo en el colegio semanalmente o incluso menos veces por semana y tener conexión a internet en el hogar.

\section{Referencias}

Almenara, J. (1996). "Nuevas tecnologías, comunicación y educación". En: Revista electrónica de tecnología Educativa, (1)

Angrist, J. \& Lavy, V. (2002). "New evidence on classroom computers and pupil learning". In: Economic Journal, 112, pp. 735-765.

Aypay, A. (2010). "Information and communication technology (ICT) usage and achievement of Turkish students in PISA 2006". TOJET, 9(2).

Banco Mundial (2009). "La calidad de la educación en Colombia: un análisis y algunas opciones para un programa de política". [En línea] Disponible en Instituto Colombiano para la Evaluación 
La influencia de las tic en el desempeño académico: Evidencia de la prueba pirls en colombia 2011

de la Educación. http://hydra.icfes.gov.co/pisa/ Documentos/ [Consultado: Octubre 17 de 2013]

Becker, G. (1964). Human Capital and the Goal Distribution of Income: An Analytical Approach. New York: Columbia University Press.

Biagi, F. \& Loi (2013). "Measuring ICT Use and Learning Outcomes: evidence from recent econometric studies". In: European Journal of Education, Vol. 48, No. 1, 2013

Borthwick, A. \& Lobo, I. (2005). Lessons from Costa Rica. Learning and Leading with Technology, 33(2), 18-21.

Buckner, et al. (2001). "Predictors of academic achievement among homeless and low-income housed children". In: Journal of School Psychology, 39(1), 45-69.

Castells, M. (1999). "Flows, Networks, and Identities: a critical theory of the information society. In: Castell et al, Critical Education in the New Information Age. Rowman y Littlefield Publishers, Inc. Lanhm.

Cenich, G. y Santos, G. (2005). "Propuesta de aprendizaje basado en proyectos y trabajo colaborativo: experiencia de un curso en línea". En: Revista Electrónica de Investigación Educativa. Vol.7, $\mathrm{n}^{\mathrm{o}} 2$, http://redie.uabc. $\mathrm{mx} /$ vol7no2/contenido-cenich.html.

Comber, C. et al. (1997). "The effects of age, gender and computer experience upon computer attitudes". In:

Educational Research, 39(2), 123-133.

Cooperberg, A.F. (2002). "Las herramientas que facilitan la comunicación y el proceso de enseñanza-aprendizaje en los entornos de educación a distancia". En: Revista de Educación a Distancia, (3).

Fernández, B.; Suárez, L. y Álvarez, E. (2006). "El camino hacia el Espacio Europeo de Educación Superior: deficiencias metodológicas y propuestas de mejora desde la perspectiva del alumno". En: Aula Abierta. $\mathrm{N}^{\mathrm{o}}$ 88, pp. 85-105.

Ferro Soto; Martínez Serna y Otero Neira (2009). "Ventajas del uso de las TICs en el proceso de enseñanza-aprendizaje desde la óptica de los docentes universitarios españoles. EDUTEC". En: Revista electrónica de tecnología educativa, (29), 5.

Foy, P. (2013). "TIMSS and PIRLS 2011 user guide for the fourth grade combined international database". TIMSS \& PIRLS International Study Center. Boston College.
Revisado 12 de enero de 2014. http://timssandpirls. bc.edu/timsspirls2011/ downloads/TP11_UserGuide. pdf

Frow, J. \& Emmison, M. (1998). "Information Technology as Cultural Capital". In: Australian Universities' Review, 41(1), 41-45.

Fuchs, T. \& Woessmann, L. (2004). "Computers and Student Learning: Bivariate and Multivariate Evidence on the Availability and Use of Computers at Home and at School". CES ifo Working Paper No.1321. Category 4: Labour Markets

García-Valcárcel, A.; Tejedor, F.J. \& Diéguez, J.L.R. (1996). Perspectivas de las nuevas tecnologías en la educación (pp. 185-199). Madrid: Narcea.

Goolsbee, A. y Guryan, J. (2006). "The impact of Internet subsidies in public schools", In: The Review of Economics and Statistics, 88, pp. 336-347.

Gorman, K. y Politt, E. (1999). "Determinants of school performance in Guatemala: Family background characteristics and early abilities". In: International Journal of Behavioral Development, 16, 75-91.

Lara, P. y Duart, J.M. (2005). “Gestión de contenidos en el e-Learning: acceso y uso de objetos de información como recurso estratégico". En: Revista de Universidad y Sociedad del Conocimiento. Vol. 2, $\mathrm{n}^{\mathrm{o}}$ 2, http://www. uoc.edu/rusc/2/2/dt/esp/lara.pdf.

Lee, Y.H., \& Wu, J.Y. (2012). "The effect of individual differences in the inner and outer states of ICT on engagement in online reading activities and PISA 2009 reading literacy: Exploring the relationship between the old and new reading literacy. Learning and Individual

Differences", 22(3), 336-342.

Legler, H.; Licht, G. \& Spielkamp, A. (2000). “Germany's Technological Performance: A Study on Behalf of the German Federal Ministry of Education and Research" (Vol. 8). Springer.

Makrakis, V. \& Sawada, T. (1996). "Gender, computers and other school subjects among Japanese and Swedish pupils". In: Computers and Education, 26(4), 225-231.

Marqués, P. (2001). "Algunas notas sobre el impacto de las TIC en la universidad”. En: Educcar. Vol. 28, págs. 83-98. 
Martínez, S.; Tarazona, A.C. y Hervas, A. (2003). "La calidad en el proceso de formación vía Internet: el planteamiento de la Universidad Politécnica de Valencia". En: III Congreso Aplicación de las Nuevas Tecnologías en la Docencia Presencial y e-Learning, Valencia.

Mata, F. (2002). "Universidad y TIC. Implicaciones prácticas”. En: Congreso Europeo de Aplicación de las Nuevas Tecnologías a la Enseñanza, Barcelona. España.

Mayer, R. (2000). "Diseño educativo para un aprendizaje constructivista". En Reigeluth, Charles (ed.), Diseño de la Instrucción. Teorías y modelos. Madrid: Aula XXI Santillana, págs. 154-171.

McFarlane, A.; Sparrowhawk, A. \& Heald, Y. (2002). "Report on the educational use of games: An exploration by TEEM of the contribution which games can make to the education process". [on line]. Available at Centre D’Educació I Noves Tecnologies: http://reservoir.cent. uji.es/canals/octeto/es/440. [consulted: march 14 of 2011].

Meelissen Martina, R.M. \& Drent, M. (2008). "Gender diferences in computer attitudes: Does the school matter?" In: Computers in Human Behavior, 24 (2008) 969-985

Notten, N. \& Kraaykamp, G. (2009). "Home media and science performance: a cross national study" In: Educational Research and Evaluation, 15, pp. 367-384.

OCDE (2010). El Informe Pisa 2006. [En línea]. Disponible en Más Actual http://www.masactual.com/pdf/ [consultado: febrero 20 de 2011]
Ronteltap, F. \& Eurelings, A. (2002). “Activity and interaction of students in an electronic learning environment for problem-based learning". In: Distance Education. Vol. 23, $\mathrm{n}^{\mathrm{o}} 1$, pp. 11-22.

Salinas, J. (1998). "Redes y educación: Tendencias en educación flexible y a distancia”. En: Pérez et ál. (coords.). Educación y Tecnologías de la Educación. Oviedo: II Congreso Internacional de Comunicación, Tecnología y Educación, http://www.uib.es/depart/gte/ tendencias.html.

(1999). "Qué se entiende por una institución de educación superior flexible”. EDUTEC'99, Universidad de Sevilla. http://www.uib.es/depart/gte/ edutec99.html.

Spiezia, V. (2010). "Does computer use increase educational achievements? Student level Evidence from PISA" In: OECD Journal of Economic Studies, Volume 2010.

Sutton, A. \& Soderstrom, I. (1999). "Predicting elementary and secondary school achievement with school-related and demographic factors". In: The Journal of Educational Research, 92, 330-338.

Volman, M. et al. (2005). "New technologies, new differences. Gender and ethnic differences in pupils' use of ICT in primary and secondary education". In: Computers and Education, 45(1), 35-55.

Wengslinky, H. (1998). "Does it compute? The relationship between educational technology and student achievement in mathematics". In Educational Testing Services (ETS) Policy Information Report. 